\title{
Applications of Weak, Complex Magnetic Fields that Attenuate EAE in Rats to a Human Subject with Moderately Severe Multiple Sclerosis
}

\author{
Michael A Persinger ${ }^{*}$, Stanley A Koren, and Linda S St Pierre
}

Clinical Neuroscience Laboratory, Behavioural Neuroscience Program, Laurentian University, Sudbury, Ontario, Canada

*Corresponding author: Clinical Neuroscience Laboratory, Behavioural Neuroscience Program, Laurentian University, Sudbury, Ontario, Canada P3E 2C6, Tel: 01-705-675-4284; Fax: 01-705-671-3844; E-mail: mpersinger@laurentian.ca

Received date: Mar21, 2014, Accepted date: June 24, 2014, Published date: June 24, 2014

Copyright: (c) 2014 Persinger MA, et al. This is an open-access article distributed under the terms of the Creative Commons Attribution License, which permits unrestricted use, distribution, and reproduction in any medium, provided the original author and source are credited.

\begin{abstract}
Background: A middle-aged woman who displayed moderately severe chronic multiple sclerosis for more than a decade and whose symptoms did not respond to conventional treatments volunteered to whole-body exposures of computer generated, $7 \mathrm{~Hz}$ weak magnetic fields during her sleep cycle for about four months.
\end{abstract}

Method: The magnetic fields were generated by custom-constructed software that generated current through two large coils that were placed at the head and foot of the bed and extended the width of the bed. To simulate the procedure that had markedly ameliorated experimental allergic encephalomyelitis in Lewis rats, the $7 \mathrm{~Hz}$ fields were presented for $6 \mathrm{~min}$ once per hour, between midnight and $08 \mathrm{hr}$. The generated maximum (peak-to-peak) intensities were either $40 \mathrm{nT}, 10 \mathrm{nT}$ or $0 \mathrm{nT}$ during different 30 to 40 day blocks.

Results: Only the somatic symptoms but not the ambulatory complications were significantly reduced during the nocturnal presentations of $40 \mathrm{nT}$ exposures compared to the other conditions.

Conclusions: These results suggest that this particular configuration of magnetic fields, which were shown to reduce the clinical severity of experimental allergic encephalomyelitis in rats, may help reduce the somatic components of patients with chronic multiple sclerosis.

Keywords: Multiple sclerosis; Experimental trial; Weak; Whole body exposures; Magnetic fields; $7 \mathrm{~Hz} ; 40 \mathrm{nT}$

\section{Introduction}

In the balance of probabilities the aggregates of overt behaviours and histological indicators now labelled as Multiple Sclerosis (MS) are likely to be a cluster of similar phenomena originating from very different etiologies. The primary consequence of assuming that MS is a homogeneous process originating from a singular source of variance is the potential for over inclusion of quite unrelated entities that respond optimally to quite different treatments or procedures. Historically the early stages of MS and variants of dissociative and psychosomatic disorders, once labelled as "hysteria", are difficult to distinguish although MS is more likely to present with paraesthesia, increased reports of fatigue, nystagmus, intermittent ankle clonus and diminished abdominal reflexes [1].

Early treatments with less harsh interventions than immunosuppressive therapies might benefit patients or at least ameliorate concomitant symptoms. The most frequent psychiatric correlates include fluctuations between depression to euphoria but without the cognitive "acceleration" typical of hypomania [2]. Intellectual correlates during the initial presentation can include memory deficits for recent events, conceptual impairments, and even dementia. When a schizophreniform pattern is present the themes are dominated by expansive, delusional or hypervigilant ("paranoid") content. The multiplicity of these symptoms and the likely multivariate nature of their origins suggest that singular pharmacological treatments restricted by vascular delivery to target tissue should be only partially effective.

Time-varying magnetic fields whose frequencies are congruent with resonance characteristics of the human brain and neuronal tissue have the capacity to penetrate cerebral space. Recently Persinger and Saroka [3] demonstrated that weak, microTesla magnetic fields penetrated organic impedances that were twice the thickness of the human skull without any obvious attenuation. We have also shown that application of microTesla transcerebral magnetic fields is associated with quantities of energy that are within the same order of magnitude as those associated with the injection of local electric currents sufficient to evoke significant experiences within surgical settings [4].

Although the validity of rodent models, particularly experimental allergic encephalomyelitis (EAE) has been sometimes questioned, the general similarity between the neurohistological features of both cannot be denied. EAE is associated with the infiltration of mononuclear cells into primarily the brain stem and cerebral (white matter) tissue of the Lewis rat. They originate from the vasculature as suggested by cell density gradients and are associated with reduced numbers of mast cells (compared to non-affected subjects). Like MS, EAE-related histological indicators can occur without obvious overt symptoms and can be exacerbated by trauma including subclinical complex partial epileptic seizures [5].

Cook and Persinger [6] reported that whole-body exposures to an amplitude-modulated ( $1 \mathrm{nT}$ to $50 \mathrm{nT}$ ) $7 \mathrm{~Hz}$ field applied through Helmholtz coils for 6 min once per hour for $8 \mathrm{hr}$ during the night significantly reduced the severity of (or even prevented) the hypotonus 
of the tails and hind limbs of rats in which experimental allergic encephalomyelitis, the rat model for multiple sclerosis, had been induced. Neither $7 \mathrm{~Hz}-500 \mathrm{nT}, 40 \mathrm{~Hz}-1 \mathrm{nT}$ to $50 \mathrm{nT}$, nor $40 \mathrm{~Hz}-500$ $\mathrm{nT}$ exposures differed significantly from non-exposed controls who developed severe manifestations of the disorder. Subsequent studies verified the importance of the intermittent but nightly durations of treatment [7]. There was also a reduction in the infiltration of mononuclear cells that was both frequency and intensity dependent [8].

There is also evidence that exposure to weak magnetic fields can be beneficial for at least some patients diagnosed with MS. Sandyk [9] reported an attenuation of the symptoms in a 50 year old female patient with multiple sclerosis following treatment with an electronic device (Ergo, Athens, Greece). This matrix of 32 coils (each having a diameter of $1 \mathrm{~cm}$ ) generated a frequency range between $2 \mathrm{~Hz}$ and $7 \mathrm{~Hz}$ with an intensity of 7.5 pico Tesla. It was applied over the vertex on the scalp for $7 \mathrm{~min}$. These intensities are more than a thousand times less than the ones employed in our rodent studies. However the intrinsic magnetic field strengths associated with whole volume neuronal activity is within this range. There is accumulating evidence that like human language where the syntax of the sentence usually contains more information than its loudness the pattern of the applied magnetic field could me more important than intensity once a threshold has been achieved.

Given these conditions, we reasoned that application of the appropriately-tuned magnetic field over the entire body of a patient who has displayed verified MS for a decade and for whom all current treatments have been exhausted could be potentially effective. If this occurred, there could be an additional option for patients who are subjected to the incapacitation of this disorder. We selected the $7 \mathrm{~Hz}$, computer generated magnetic field whose software was identical to the field generated through Helmholtz coils by another type of computer application for the rodent experiments. If the laboratory rodent experiments can be generalized, then the intermittent nocturnal magnetic field application at $40 \mathrm{nT}, 7 \mathrm{~Hz}$ should be most effective compared to less intense applications. The Jacobson-Persinger model for energy (rather than force-related) resonance [10] indicates the solution for a $40 \mathrm{nT}$ field is congruent with affecting a molecule with a mass equivalent to melatonin.

We calculated that the intracerebral effects of the $7 \mathrm{~Hz}$ field would be additionally enhanced for two reasons. First, the resonance of the cerebral circumference assuming an approximately $60 \mathrm{~cm}$ value and the bulk velocity of the rostral-caudal cortical neurons to be about 4.5 $\mathrm{m} \cdot \mathrm{s}^{-1}$ is about $7.5 \mathrm{~Hz}$. Second, the inductance (L) per m (permeability) of cortical grey matter at $1 \mathrm{kHz}$ is about $10^{-2}$ Henrys. The permittivity (C) of grey matter is $\sim 2.5 \cdot 10^{-1}$ Farads $\cdot \mathrm{m}^{-1}$. We assumed $\sim \mathrm{kHz}$ because the rise time for the point durations for the port times from the computer were within this range. Application of the formula: $f=(2 \pi \cdot \sqrt{ }$ (LC) $1 / 2)^{-1}$, the equation for the resonance frequency of a circuit, results in a value of $\sim 7 \mathrm{~Hz}$ [11]. We also assumed that if the results of our experiments and our basic assumptions about the operations of complex magnetic fields were valid, then simulation of the experimental conditions within a much larger spatial dimension for a human being should also reduce the symptoms of MS.

\section{Methods and Materials}

During the course of our clinical practice in neuropsychology, a middle-aged woman whose husband had been assessed by us for a brain injury sustained during a motor vehicle incident learned about our research and volunteered to be a subject. The woman had developed the symptoms of multiple sclerosis about 18 years before the treatment began. Following the same motor vehicle incident her symptoms worsened and she required a walker for ambulation. After signing of consent documentation, the equipment and monitoring devices were moved into her bedroom.

One of two $1.5 \mathrm{~m} \mathrm{x} 1.5 \mathrm{~m}$ rectangular coils was placed at each end of the patient's bed. As a result the direction of the field generated between the two coils was along the sleeper's major axis. The length of the bed was about $2.4 \mathrm{~m}$ and the width was about $1.5 \mathrm{~m}$. It was oriented in a north-west direction. There were no headboards and the contribution from the metal base upon which the mattress rested did not produce any significant static magnetic field anomalies over the central two-thirds of the bed's surface area where the patient slept. The resultant geomagnetic field averaged about $63,500 \mathrm{nT}$ over the surface of the sleeping area.

The magnetic field was generated by software (Complex) from an $\mathrm{XT}$ computer that was placed in the far corner of the bedroom. The specific shape and wave form have been published elsewhere [12]. It was programmed to run continually and to produce the $7 \mathrm{~Hz}$, amplitude-modulated field for 6 min once per hour between midnight and $0800 \mathrm{hrs}$. The activation of the fields through the computer could not be heard by the experimenters. The patient was partially deaf. The point durations were set at $69 \mathrm{~ms}$ so that the intrinsic frequency would be square-wave $7 \mathrm{~Hz}$ oscillations upon which the amplitude modulations were superimposed.

The sequence was delivered through a custom-constructed digitalto-analogue converter to the two coils. A log program recorded the date and time of each presentation of the sequence. There were no power failures or disruptions during the exposures. To insure fidelity of the signal a commercial (Radio Shack) telephone recorder microphone (placed under the bed) was attached to a voice activated tape-recorder. The coil within the microphone was easily activated by the magnetic field and silently registered the acoustic equivalent of the changes in the amplitude of the magnetic pattern. Every second or third day the tape was recovered and played to ensure the field had been presented.

The software also controlled the amplitude of the applied field. During the first 46 days of the experiment, the field strengths over the sleeping area ranged between 1 and 40 nanoTesla. We began the treatment after four days of placing the equipment, hence we did not collect what we had planned to be a baseline, to honour the request of the patient who was experiencing substantial somatic distress. For the next 30 days the field strength was set for a maximum of $10 \mathrm{nT}$. The 40 $\mathrm{nT}$ (peak) intensity was instituted for another 30 days. Finally during the last 34 days of the experiment the cables to the coils were disconnected (without the patient's knowledge).

The patient usually retired for sleep about 22 to $23 \mathrm{hrs}$ each night and completed the sleep cycle about $08 \mathrm{hrs}$. Consequently, for most nights she was exposed to the 8,6 min periods of the nocturnal magnetic fields that were generated along her major axis while she was sleeping. At the end of each day the patient employed a 0 (no change) or 1 (presence of symptom or worsening of symptoms) scale to rate the domains of somatic pain, ambulation difficulties, or headache.

After 144 days of the experiment, following discussions with family members about the treatment, the patient requested removal of the equipment. As agreed within the consent form, the reasons for the 
termination of the treatment at any time were neither required nor requested by the experimenters. The data from the diary were then analyzed.

\section{Results}

The percentage of days during which the symptoms were present for somatic symptoms (primarily pain in the limbs) were $11 \%, 40 \%$, $3 \%$, and $32 \%$ for the $40 \mathrm{nT}, 10 \mathrm{nT}, 40 \mathrm{nT}$, and 0 (coils disconnected) $\mathrm{nT}$ conditions, respectively. Chi-squared analyses indicated that the proportions were significantly different [chi-squared $(\mathrm{df}=2)=18.29, \mathrm{p}$ $<.001$ ]. The phi (correlation coefficient equivalent) was $0.36(\mathrm{p}<.001)$. When the $40 \mathrm{nT}$ field was present the likelihood that the patient reported somesthetic complaints the following day was $0.14(95 \%$ confidence interval=.05 to 0.38 ) compared to days following exposure to either the no field or $10 \mathrm{nT}$ field intensity.

A series of post hoc contingency tables indicated that the primary source of the statistical significance was due to the markedly reduced proportion of somatic symptoms during the period she slept in the 40 $\mathrm{nT}$ fields compared to the $10 \mathrm{nT}$ and sham field whose scores did not differ significantly from each other. There were no significant differences in the proportions of days that ambulation was more difficult or headaches were more prominent

According to the patient's diary, the first symptom that was different from the week before the experiment began to occur about four days after commencement of the experimental protocol (40 nT field). She reported the experience of an extreme numbness in the right hand that was attributed to the right hand; the duration was for about $1 \mathrm{~min}$. Sinus headaches were recorded on the fifth and sixth days after the initiation of the experiment while chest pains before retiring for the night (about $2330 \mathrm{hrs}$ ) were noted. However, after the 10th day the patient reported she could lift her left arm and left leg more easily while lying in bed. Sleep was considered "better" by both her husband and herself for the next month.

During the period when the field strength was decreased her sleep was considered more disturbed. The left leg and arm were more difficult to bend and move. During the second introduction of the 40 $\mathrm{nT}$ fields sleep improved according to her reports. Sinus headaches were reported during the first few days of this interval. She reported experiencing more fatigue. During the course of the experiment there were no conspicuous events, e.g., trauma, in the patient's life that would have confounded the treatment.

\section{Discussion}

The results of this study partially support the hypothesis that the same complex configuration of nocturnal magnetic fields at strengths around $40 \mathrm{nT}$, the same intensity that markedly reduced overt symptoms of EAE in Lewis rats [6] and was the optimal intensity for the energy resonance equation [10], can improve the symptoms of multiple sclerosis at least in one patient. Less intense field strengths did not attenuate the proportions of somatic symptoms. A second presentation of the $40 \mathrm{nT}$ field, after about one month of a less intense field, reduced the symptoms as well. When the fields were inactivated totally, the proportions of reports increased markedly.

Cook and Persinger [6] found that the $7 \mathrm{~Hz}, 40 \mathrm{nT}$ field employed in this study also reduced the clinical symptoms of experimental allergic encephalomyelitis in Lewis rats while stronger fields (500 nT) or weaker fields (less than $10 \mathrm{nT}$ ) were not effective. That frequency is important was demonstrated as well. When the $40 \mathrm{nT}$ field was applied as $40 \mathrm{~Hz}$ rather than $7 \mathrm{~Hz}$ fundamentals, there was no amelioration of the symptoms. The behaviorally effective strength and frequency also reduced the numbers of infiltrations of mononuclear cells into the parenchyma of the brain stem and diencephalon [7].

However the current treatment did not change the subjective ratings of improvement for ambulation for this patient. The improvement that was present occurred for the left leg and left arm while the patient was laying but not walking. It may be of interest that Sandyk [9] has reported that application of weaker but more complex magnetic fields over the right hemisphere has improved clinical symptoms. Whether or not the right hemisphere is particularly responsive to the beneficial effects of the appropriately patterned magnetic field must still be investigated.

The proportion of mast cells in rats developing EAE while exposed to this protocol were more affected within the right side of the brain than the left $[7,13]$. There is the possibility that the apparent right hemispheric sensitivity may be confounded by the approximately $10 \%$ greater "blood flow" and proportion of white matter than in the typical left hemisphere. However, recent quantitative electroencephalographic measurements for large populations indicated that right hemispheric activity within the theta range is correlated with increases in global geomagnetic activity [14,15].

In our opinion the results of this study are sufficient to encourage more organized clinical trials. Modern computer equipment and software, as well as the ease of constructing the coils, would require minimal expense. Nocturnal exposure while the patient sleeps is also minimally intrusive and allows a more or less consistent exposure protocol. Verification of field presence is easily completed. At the very least the procedure could allow some relief from somatic symptoms.

The major impedance is more likely to be from well-intended contributors to the patient's decisions. Later we found that the primary reason this patient decided to stop the treatment was because one of her children, who knew a medical health professional, convinced the patient that any such procedure was "unscientific" and could have adverse health effects. Establishing face-validity and education of the patient, family, and health professionals may be as challenging as the technical aspects of the exposure protocol.

\section{References}

1. Golden CJ, Moses JA, Coffman JA, Miller WR, Strider FD (1983) Clinical Neuropsychology: Interface with Neurologic and Psychiatric Disorders. Grune\& Stratton, N.Y.

2. Cummings HL (1985) Clinical Neuropsychiatry. Grune\& Stratton, N.Y.

3. Persinger MA, Saroka KS (2013) Minimum attenuation of physiologically-patterned, 1 microTesla magnetic fields through simulated skull and cerebral space. J ElectromagAnalys App 5: 151-155.

4. Persinger MA, Saroka KS (2013) Comparable portions of classes of experiences and intracerebral consequences for surgical stimulation and external application of weak magnetic field patterns: implications for converging effects in complex partial epileptic experiences. EpilepBehav 27: 220-224.

5. Cook LL, Persinger MA (1999) Infiltration of lymphocytes in the limbic brain following stimulation of subclinical cellular immunity and low dosages of lithium and a cholinergic agent. ToxicolLett 109: 77-85.

6. Cook LL, Persinger MA (2000) Suppression of experimental allergic encephalomyelitis is specific to the frequency and intensity of nocturnally applied, intermittent magnetic fields in rats. NeurosciLett 292: 171-174. 
Citation: Persinger MA, Koren SA, St Pierre LS (2014) Applications of Weak, Complex Magnetic Fields that Attenuate EAE in Rats to a Human Subject with Moderately Severe Multiple Sclerosis. J Neurol Neurophysiol 5: 213. doi:10.4172/2155-9562-5-1000213

Page 4 of 4

7. Kinoshameg SA, Persinger MA (2004) Suppression of experimental allergic encephalomyelitis in rats by $50-\mathrm{nT}, 7-\mathrm{Hz}$ amplitude-modulated nocturnal magnetic fields depends on when after inoculation the fields are applied. NeurosciLett 370: 166-170.

8. Persinger MA (2009) Differential numbers of foci of lymphocytes within the brains of Lewis rats exposed to weak complex nocturnal magnetic fields during development of experimental allergic encephalomyelitis. Int J Neurosci 119: 166-184.

9. Sandyk R (1992) Successful treatment of multiple sclerosis with magnetic fields. Int J Neurosci 66: 237-250.

10. Persinger MA (2004) A potential multiple resonance mechanism by which weak magnetic fields affect molecules and medical problems: the example of melatonin and experimental â€œmultiplesclerosisâ€. Med Hypoth 66: 811-815.

11. Persinger MA (2012) Brain electromagnetic activity and lightning: potentially congruent scale-invariant quantitative properties. Front IntegratNeurosci 6: 19.
12. Murugan NJ, Karbowski LM, Lafrenie RM, Persinger MA (2013) Temporally-patterned magnetic fields induce complete fragmentation in planaria. PLoS One 8: e61714.

13. Cook LL, Persinger MA, Koren SA (2000) Differential effects of low frequency, low intensity $(<6 \mathrm{mG})$ nocturnal magnetic fields upon infiltration of mononuclear cells and numbers of mast cells in Lewis rat brains. ToxicolLett 118: 9-19.

14. Mulligan BP, Persinger MA (2012) Experimental simulation of the effects of sudden increases in geomagnetic activity upon quantitative measures of human brain activity: validation of correlational studies. NeurosciLett 516: 54-56.

15. Saroka KS, Caswell JM2, Lapointe A3, Persinger MA4 (2014) Greater electroencephalographic coherence between left and right temporal lobe structures during increased geomagnetic activity. NeurosciLett 560: 126-130 